\title{
Perturbative Machine Learning Technique for Nonlinear Impairments Compensation in WDM Systems
}

\author{
Evgeny Averyanov ${ }^{(1,2)}$, Alexey Redyuk ${ }^{(2,1)}$, Oleg Sidelnikov $v^{(1,2)}$ \\ Mariia Sorokina $^{(3)}$, Mikhail Fedoruk ${ }^{(1,2)}$, Sergei Turitsyn ${ }^{(1,3)}$
}

\author{
(1) Novosibirsk State University, Novosibirsk, 2 Pirogova str., 630090 Russia \\ (2) Institute of Computational Technologies SB RAS, Novosibirsk, 6 acad. Lavrentjev av., 630090 Russia, \\ alexey.redyuk@gmail.com \\ (3) Aston Institute of Photonic Technologies, Aston University, B4 7ET Birmingham, UK
}

\begin{abstract}
We propose a perturbation-based receiver-side machine-learning equalizer for inter- and intra-channel nonlinearity compensation in WDM systems. We show $1.6 \mathrm{~dB}$ and $0.6 \mathrm{~dB} Q^{2}$-factor improvement compared with linear equalization and DBP respectively for $1000 \mathrm{~km}$ transmission of 3x128Gbit/s DP-16QAM signal.
\end{abstract}

\section{Introduction}

Nonlinear transmission impairments are one of the key factors limiting further improvements of the modern optical-fibre communication systems to operate at higher information transmission rates and larger reach. To tackle this problem, a number of techniques have been proposed. Among them, digital back-propagation ${ }^{1}$ (DBP), Volterra function based methods ${ }^{2}$, perturbationbased pre-distortion and perturbation-based post-equalization techniques ${ }^{3,4}$ (PPD/PPE). However, practical implementation of these methods is still facing a number of challenges. The inter-channel nonlinearity compensation is especially important in the wavelength-division multiplexing (WDM) coherent transmission systems. The application of compensation methods in real-time mode potentially can be achieved with the help of machine learning techniques, fast developing in the field of optical communications the past few years ${ }^{5}$.

In the paper ${ }^{6}$ authors reviewed the modeling of inter-channel nonlinear interference noise considering the interaction of two channels with two polarizations. To estimate these interactions one need to calculate thousands of the perturbation coefficients given by multidimensional overlapping integrals, the number of which increases proportionally to the number of channels.

As was proposed in ${ }^{7}$, instead of numerical integration, one can employ robust regression methods to identify perturbation coefficients in one step. This approach has been used in single- and multi-channel space-division multiplexed systems and enabled high efficiency with relaxed complexity ${ }^{7}$.
In this paper we apply this method for wavelength-division-multiplexed (WDM) channels for compensating intra- and inter-channel nonlinear effects, using information from neighbour channels to restore original signal. The method enables $1.6 \mathrm{~dB}$ and $0.6 \mathrm{~dB}$ improvement in $Q^{2}$ factor compared to linear equalization and ideal one-channel DBP, respectively.

\section{Perturbation-based nonlinear compensation}

A first order perturbation analysis of the Manakov equations yields an expression for nonlinear three-pulse interactions of different polarizations and channels, which can be written as $X_{k}^{s}=$ $x_{k}^{s}+\Delta x_{k}^{s}$, where $x_{k}^{s}$ and $X_{k}^{s}$ are complex amplitudes in time slot $k$ in $x$-polarization in channel $s$ in transmitter and receiver, respectively. Then nonlinear distortions in channel $s$ in time slot $k$ is given by Eq. (1)

$$
\begin{gathered}
\Delta x_{k}^{s}=\sum_{m, n} C_{m, n}\left(x_{k+m}^{s} x_{k+n}^{s} \bar{x}_{k+m+n}^{s}+\right. \\
\left.x_{k+m}^{s} y_{k+n}^{s} \bar{y}_{k+m+n}^{s}\right)+ \\
\sum_{c \neq s m, n} \sum_{m, n} D_{m+n}^{c}\left(2 x_{k+m}^{s} x_{k+n}^{c} \bar{x}_{k+m+n}^{c}+\right. \\
\left.x_{k+m}^{s} y_{k+n}^{c} \bar{y}_{k+m+n}^{c}+y_{k+m}^{s} x_{k+n}^{c} \bar{y}_{k+m+n}^{c}\right),
\end{gathered}
$$

where $C_{m, n}$ and $D_{m, n}^{c}$ are the symbol triplet perturbation coefficients. In trivial cases there are analytic expressions to calculate these coefficients, however in general it requires a numerical and timeconsuming integration. Nonlinear interactions for $y$-polarization amplitudes $y_{k}^{s}$ can be obtained form Eq. (1) by replacing $x$ symbols with $y$ symbols and vice versa. 


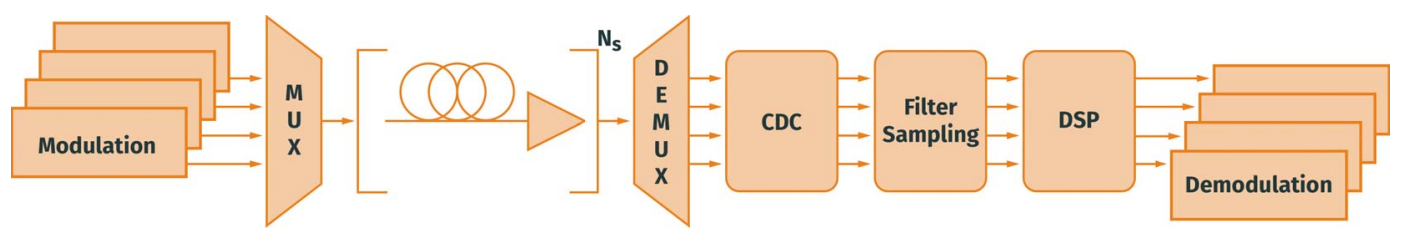

Fig. 1: Principle scheme of transmission line

The main idea of the proposed method is identifying of perturbation coefficients $C_{m, n}$ and $D_{m, n}^{c}$, applying machine learning methods to the training set of known transmitted $x_{k}^{s}$ and received $X_{k}^{s}$ symbols. After learning defined coefficients are used to compensate for nonlinearity, applying it to the test set of received symbols $X_{k}^{s}$ using expression

$$
x_{k}^{s}=X_{k}^{s}-\Delta x_{k}^{s} .
$$

The major obstacle to direct utilization of Eq. (2) on receiver-side is the need to know transmitted amplitudes $x_{k}^{s}$ and $y_{k}^{s}$ in advance in order to calculate $\Delta x_{k}^{s}$. Here we propose to take received symbols $X_{k}^{s}$ after linear phase-shift equalizer (PSE) as approximation of transmitted symbols $x_{k}^{s}$ so that it is possible to use PPE. Another approach is to perform hard-decision detection of the received symbols $X_{k}^{s}$ and use it as approximation of transmitted symbols $x_{k}^{s}$. These two approaches we called PPE(PSE) and PPE(HD), respectively.

\section{Transmission system model}

To measure performance of proposed method numerical experiments were performed. Principle scheme of transmission line is shown on Fig. 1 and all parameters summarised in Tab. 1. Under consideration was 1-channel and 3-channel 1000 $\mathrm{km}$ transmissions of dual-polarisation 16QAM signal. The transmission consisted of RRC pulses at 32 GBaud and channel spacing of $35 \mathrm{GHz}$. Number of transmitted symbols for training and test run for each polarization was $2^{14}$. Lumped amplification scheme was used with span length 100 $\mathrm{km}$ and EDFA noise figure $4.5 \mathrm{~dB}$. At the receiver after demultiplexing channels we perform ideal chromatic dispersion compensation, matched filter sampling and digital signal processing. Additionally we assumed ideal carrier phase and frequency estimation.

Signal propagation in our model governed by Manakov equations:

$$
\frac{\partial A_{x, y}}{\partial z}=\left(-\frac{\alpha}{2}-i \frac{\beta_{2}}{2} \frac{\partial^{2}}{\partial t^{2}}+i \gamma \frac{8}{9}\left[\left|A_{x}\right|^{2}+\left|A_{y}\right|^{2}\right]\right) A_{x, y},
$$

where $A_{x, y}$ is the complex field envelopes for $x$ and $y$ polarizations, $\alpha$ is the attenuation coeffi-
Tab. 1: Numerical model parameters

\begin{tabular}{|c|c|}
\hline Distance & $10 \times 100 \mathrm{~km}$ \\
\hline Bandwidth & $32 \mathrm{GBaud}$ \\
\hline RRC roll-off & 0.01 \\
\hline Wavelength & $1550 \mathrm{~nm}$ \\
\hline Noise Figure & $4.5 \mathrm{~dB}$ \\
\hline Attenuation & $0.2 \mathrm{~dB} / \mathrm{km}$ \\
\hline Dispersion & $17 \mathrm{ps} / \mathrm{nm} / \mathrm{km}$ \\
\hline Nonlinearity & $1.41 / \mathrm{W} / \mathrm{km}$ \\
\hline
\end{tabular}

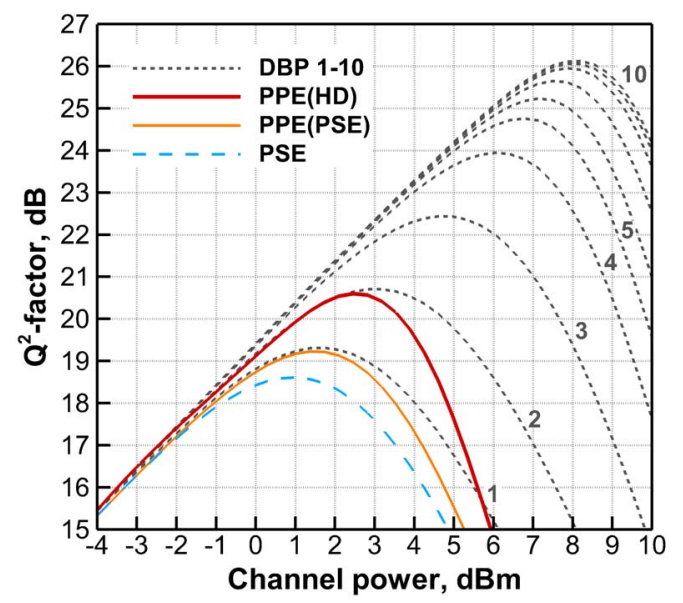

Fig. 2: One channel transmission

cient, $\beta_{2}$ and $\gamma$ are dispersion and Kerr coefficients, respectively. Numerical simulation was performed with a typical symmetrized split-step Fourier method with 16 oversampling factor.

\section{Numerical results}

To evaluate the effectiveness of the proposed scheme, it was compared with the linear equalizer PSE, that only recovers the phase of the received signal, and with the digital back-propagation algorithm with a different number of steps per span.

Fig. 2 shows the $Q^{2}$-factor as a function of initial average power for single channel transmission with different nonlinear impairments compensation schemes. It can be noted that the proposed methods provide performance at DBP1 (1 step per span) and DBP-2 level. Moreover the PPE $(H D)$ method shows the $Q^{2}$-factor higher than PPE(PSE), based on linear phase recovery method. This can be explained by the fact that the HD approach can approximate the transmitted signal better then PSE. It should also be noted that the obtained results are consistent with 


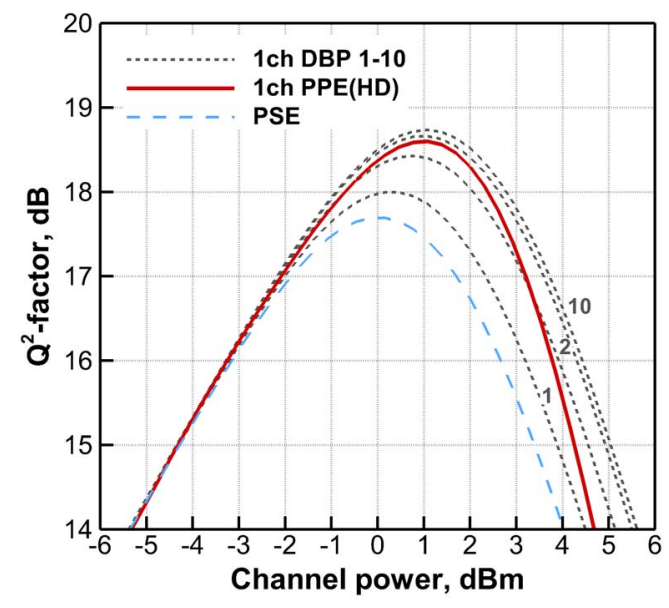

Fig. 3: Three channel transmission

the single-mode transmission case of results presented recently ${ }^{7}$.

We also tested the proposed scheme in the case of 3-channel WDM transmission. Fig. 3 shows the $\mathrm{Q}^{2}$-factor of central channel as a function of initial power per channel. Here to compensate for nonlinear impairments we use information only from the central channel. In this case at an optimum power the approach based on harddecision now shows the $Q^{2}$-factor only slightly less than the converged DBP-5 (i.e. the further increase in the number of steps per span does not lead to an increase in the $Q^{2}$-factor). It should also be noted that with the same performance the proposed method requires much less computational operations than the DBP with 5 steps per span.

Finally we considered the case when information from all channels is used for nonlinear equalization (see Fig. 4). As expected, PPE(HD) using three channels provides better performance

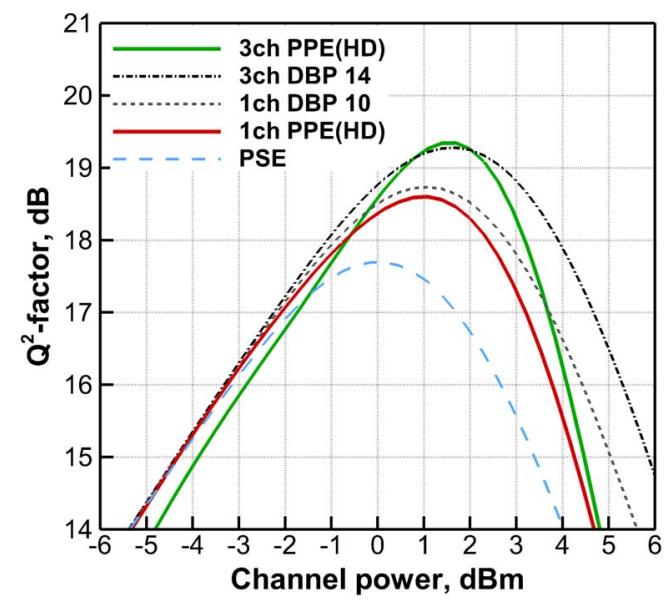

Fig. 4: Three channel transmission improvement then PPE $(\mathrm{HD})$ for only one channel. Moreover, in this case the proposed method shows the $\mathrm{Q}^{2}$-factor improvement of $1.6 \mathrm{~dB}$ in comparison with PSE and surpasses full-loaded DBP with 14 steps per span. Note that observed $1.6 \mathrm{~dB} \mathrm{Q} \mathrm{Q}^{2}$-factor improvement for $3 \mathrm{WDM}$ channels is comparable with corresponding improvement for 3 SDM channels in ${ }^{7}$. It should also be noted that PPE(HD) shows $0.6 \mathrm{~dB}$ performance improvement compared with ideal one-channel DBP.

\section{Conclusions}

In this work we investigated a receiver-side perturbation-based method for intra- and interchannel nonlinear effects compensation. We compared performance of the proposed technique with both a linear phase equalizer and digital back propagation. In case of multichannel transmission we show $1.6 \mathrm{~dB}$ and $0.6 \mathrm{~dB}$ performance improvement compared with linear equalization and ideal one-channel DBP.

\section{Acknowledgements}

The work was supported by the Russian Science Foundation (Grant No. 17-72-30006).

\section{References}

[1] E. Ip, "Nonlinear Compensation Using Backpropagation for Polarization-Multiplexed Transmission,“ J. Lightwave Technol., Vol. 28, no. 6, pp. 939-951, (2010).

[2] L. Liu et al., "Intrachannel Nonlinearity Compensation by Inverse Volterra Series Transfer Function," J. Lightwave Technol., Vol. 30, no. 3, pp. 310-316 (2012).

[3] T. Oyama et al., "Robust and efficient receiver-side compensation method for intra-channel nonlinear effects," OFC, Tu3A.3, San Francisco (2014).

[4] Z. Tao et al., "Multiplier-Free Intrachannel Nonlinearity Compensating Algorithm Operating at Symbol Rate," J. Lightwave Technol., Vol. 29, no. 17, pp. 2570-2576 (2011).

[5] D. Zibar et al., "Machine Learning Techniques in Optical Communication," J Lightwave Technol, Vol. 34, no. 6, pp. 1442-1452 (2016).

[6] R. Dar et al., "Inter-Channel Nonlinear Interference Noise in WDM Systems: Modeling and Mitigation," J. Lightwave Technol, Vol. 33, no. 5, pp. 1044-1053 (2015).

[7] M. Sorokina et al., "Sparse identification for nonlinear optical communication systems: SINO method," Opt. Express, Vol. 24, pp. 30433-30443 (2016). 